Authors

Jun Ye, Steven T. Cundiff, Seth M. Foreman, John L. Hall, K. W. Holman, David J. Jones, J. D. Jost, Henry C. Kapteyn, K.A.H. V. Leeuwen, Long-Sheng Ma, Margaret M. Murnane, J. L. Peng, and Robert K. Shelton 
J. YE

S.T. CUNDIFF

S. FOREMAN

T.M. FORTIER

J.L. HALL

K.W. HOLMAN

D.J. JONES

J.D. JOST

H.C. KAPTEYN

K.A.H. V. LEEUWEN

L.S. MA

M.M. MURNANE

J.L. PENG

R.K. SHELTON

\section{Phase-coherent synthesis of optical frequencies and waveforms}

JILA, National Institute of Standards and Technology and University of Colorado, Department of Physics, University of Colorado, Boulder, CO 80309, USA

\section{Received: 24 September 2001/ \\ Revised version: 30 October 2001 \\ Published online: 27 June 2002 • (C) Springer-Verlag 2002}

ABSTRACT Precision phase control of an ultrawide-bandwidth optical-frequency comb has produced remarkable and unexpected progress in both areas of optical-frequency metrology and ultrafast optics. A frequency comb (with $100 \mathrm{MHz}$ spacing) spanning an entire optical octave $(>300 \mathrm{THz})$ has been produced, corresponding to millions of marks on a frequency "ruler" that are stable at the $\mathrm{Hz}$ level. The precision comb has been used to establish a simple optical clock based on an optical transition of iodine molecules, providing an rf clock signal with a frequency stability comparable to that of an optical standard, and which is superior to almost all conventional rf sources. To realize a high-power cw optical frequency synthesizer, a separate, widely tunable single-frequency cw laser has been employed to randomly access the stabilized optical comb and lock to any desired comb component. Carrier-envelope phase stabilization of few-cycle optical pulses has recently been realized. This advance in femtosecond technology is important for both extreme non-linear optics and optical-frequency metrology. With two independent femtosecond lasers, we have not only synchronized their relative pulse timing at the femtosecond level, but have also phase-locked their carrier frequencies, thus establishing phase coherence between the two lasers. By coherently stitching the optical bandwidth together, a "synthesized" pulse has been generated with its 2nd-order autocorrelation signal displaying a shorter width than those of the two "parent" lasers.

PACS 06.20; 06.30; 42.62.-b

\section{1}

\section{Introduction}

The recent unification of ultra-precision laser technology with ultrafast lasers and non-linear optics has enabled revolutionary progress in both areas, which has significant potential for diverse applications in physical science and

Fax:+1-303/492-5235, E-mail: Ye@jila.colorado.edu technology. Of course, this explosion of interdisciplinary research activities comes to fruition only because of physical insight $[1,2]$ and mature technologies developed by many pioneers who have devoted decades of hard work in the independent fields of ultrasensitive spectroscopy, ultrastable lasers, ultrafast lasers and non-linear optics. For example, commercially available solid-state Ti:sapphire lasers [3,4] provide simple and reliable solutions to the needs of ultrafast laser technology for precision frequency metrology. The development of new optical fiber technologies [5] has allowed simple generation of octave-spanning bandwidths [6]. At present, optical frequency measurements have been reduced to a simple task, while even the highest level of measurement precision has been achieved [7-11]. Clockwork based on optical transitions has been realized $[12,13]$. Control of the carrier-envelope phase has become possible [14,15]. Pulse trains from independent mode-locked lasers have been synchronized below the $10 \mathrm{fs}$ level and their carrier waves phaselocked, leading to coherent pulse synthesis $[16,17]$. We now appear to have all the experimental tools required for complete control over coherent light, including the ability to generate pulses with arbitrary shape [18], and precisely controlled frequency and phase, and to synthesize coherent light from multiple sources. The combination of this ability to do arbitrary waveform synthesis in the optical region of the spectrum, with recently developed optical pulse measurement techniques $[19,20]$, is analogous to the development of oscilloscopes and waveform generators in the early to mid 20th century.

To understand the connection between the ultrastable and the ultrafast, we need to first point out each of their unique features. The ultrastable field is typified by the variety of highresolution spectroscopy and high-precision measurements carried out by continuous wave (cw) lasers that can be best described by their near-delta-function frequency spectra. In sharp contrast, the field of ultrafast phenomena encompasses the study of sub-picosecond events utilizing laser pulses that approach the limit of time-domain delta functions. In fact, at this point in time, state-of-the-art laser sources from these two 
fields share nearly the same delta-function "figure of merit" with frequency and temporal widths on the order of a few parts in $10^{15} \mathrm{~Hz}$ and seconds, respectively. The connection between the ultrastable and the ultrafast arises from the fact that femtosecond (fs) laser oscillators produce pulses in a periodic train via mode locking, with a corresponding rigorous periodicity in the spectral domain. In fact, the frequency-domain spectrum consists of a comb of discrete modes separated by the repetition frequency $f_{\text {rep }}=1 / \tau_{\text {r.t. }}$, where $\tau_{\text {r.t. }}$ is the cavity round-trip time. The extent of the time-domain pulse train provides the frequency resolution of individual comb components, while the total extent of the frequency-domain mode comb is approximately limited to the inverse of the pulse duration. The generation of ultrashort pulses requires that the group velocity $\left(v_{\mathrm{g}}\right)$ dispersion inside the laser cavity is minimized across the pulse frequency spectrum. This criterion is not directly related to the frequency comb spacing, since the individual mode frequencies correspond to eigenmodes of the phase-velocity $\left(v_{\mathrm{p}}\right)$ of the light. In general, we have $v_{\mathrm{g}} \neq v_{\mathrm{p}}$, due to laser cavity dispersion. This fact results in a pulse envelope function that is not fixed with respect to the underlying optical oscillation frequencies - there is a phase slip (denoted by $\Delta \phi$ ) between the "carrier" phase and the envelope peak for each of the successive pulses emitted by the laser. In the frequency domain, $\Delta \phi$ yields an offset of the mode comb from the exact harmonics of the $f_{\text {rep }}$ by the amount $f_{\text {ceo }}=\Delta \phi f_{\text {rep }} / 2 \pi$. Hence, each optical comb frequency is effectively given by $f_{n}=n f_{\text {rep }}+f_{\text {ceo }}$. Here $n$ represents an integer $\left(\sim 3 \times 10^{6}\right)$ harmonic number of the optical comb line relative to the repetition rate, $f_{\text {rep }}$.

One sees how the frequency-domain control of both $f_{\text {rep }}$ and $f_{\text {ceo }}$ makes it possible to establish a fs-laser-based optical comb at a high precision for optical frequency measurement and distribution. The bandwidth of a fs-laser comb already covers more than $100 \mathrm{~nm}$, and it can be further extended to cover an entire optical octave using non-linear optical effects enabled by microstructure fibers or other means. Subsequently, control of the pulse-to-pulse phase slip $\Delta \phi$ between the carrier and the envelope also becomes possible.

Such precise control of just a few oscillations of a highintensity laser field should dramatically impact the field of extreme non-linear optics [21], including above-threshold ionization and high-harmonic and X-ray generation with such pulses.

2

\section{Optical frequency measurement, synthesis, and distribution}

Absolute measurement of optical frequencies was historically a difficult task and required complicated multistage frequency chains for bridging vast frequency gaps [22]. A large-bandwidth optical comb permits great simplifications in optical frequency measurement since large frequency gaps can now be easily connected with various comb components and be expressed as a product of an integer number and the repetition frequency, plus a certain offset frequency. Further simplification is achieved when the comb bandwidth is extended over an entire optical octave via effective nonlinear interaction enabled by microstructure fibers [6]. With an octave-spanning spectrum, it becomes possible to meas- ure optical frequencies in a single step with a direct reference to the present realization of the SI unit of time, namely the cesium microwave standard. This is accomplished when the mode spacing $\left(f_{\text {rep }}\right)$ of the femtosecond comb is locked with feedback control to an rf signal source that is itself referenced to cesium. The octave interval between the frequency $f$ and its second harmonic $2 f$ can be simply expressed as $2 f-f=f=n f_{\text {rep }}$. This strategy can be implemented in two equivalent ways. The first approach is to use the comb to measure the frequency gap between a cw laser and its second harmonic. The second approach is to frequency-double the infrared portion of the comb spectrum and to heterodyne it with the existing visible portion of the spectrum. The resulting beat frequency is the comb offset, $f_{\text {ceo }}$. When the laser is controlled in such a fashion that both the radio frequencies $f_{\text {rep }}$ and $f_{\text {ceo }}$ are established with reference to the cesium standard, one then has an entire array of optical frequencies with precisely known frequencies $f_{n}=n f_{\text {rep }}+f_{\text {ceo }}$.

Measurement of optical frequencies has thus become a rather straightforward task. However, it was soon found that the short-term stability of the Cs clock became the limiting factor in the determination of the optical frequencies via a Cs-referenced comb [23]. This is true despite the fact that some of the optical standards used in the measurement are a few orders of magnitude more stable than the traditional Cs standard at timescales of up to an hour. Of course, there are more stable microwave signal sources available, such as hydrogen maser or Cs-fountain clock [9], but with limited availability for general use. Therefore it becomes advantageous to use an in-house optical frequency standard instead of a microwave reference to stabilize an entire optical comb. In doing so, one can in fact derive a microwave signal from the optical standard, leading to a so-called optical atomic clock. For this purpose it is important to establish an optical comb with excellent phase coherence among its individual components. The phase stability needs to exceed that of the optical standards. With this capability, we will be able to transfer the stability of a single optical oscillator to the entire comb set over its vast bandwidth, and also derive clock signals in the microwave/rf domain without any compromise in stability. Optical standards based on single ions and cold atoms promising potential stability around $1 \times 10^{-16}$ at $1 \mathrm{~s}$ [24] and potential accuracy of $1 \times 10^{-18}$ [25] may very well become future national standards, but such systems would require elaborate designs. In contrast, excellent candidates in cell-based optical frequency standards do exist, such as an iodine-stabilized Nd:YAG laser [26], that would offer compact, simple, and less expensive system configurations, albeit at the cost of performance degradation by perhaps two decades. Along with optical combs, a competent laboratory would be able to realize a network of microwave and optical frequencies at a level of stability and reproducibility that surpasses the properties of basically all normal, commercially available frequency sources, but at a reasonable cost. Easy access to the resolution and stability offered by optical standards would greatly facilitate application of frequency metrology both to precision experiments for fundamental physics and to practical devices.

Here, we demonstrate a particular realization of precision phase control of the (100 MHz fs-laser based) optical comb. Using an orthogonal control approach, we have achieved Hz- 
level $\left(\leq 3.5 \times 10^{-15}\right)$ phase-tracking stability across the entire optical octave. Furthermore, the orthogonalization procedure permits independent control of both degrees of freedom associated with the optical comb, leading to a clockwork mechanism using only one comb parameter [13]. Recent work [12] uses a two-parameter control scheme to transfer the stability of a cold-ion-based optical standard to the comb lines at the $3 \times 10^{-16}$ level. Clearly, with a mature technical solution to the "gearbox problem" at hand, all future progress in opticaldomain and rf-domain standards can be utilized in both spectral ranges.

The two variables of the comb can be expressed as: $f_{\text {rep }}=$ $v_{\mathrm{g}} / l_{\mathrm{c}}$ and $f_{\text {ceo }}=v_{0}\left(1-v_{\mathrm{g}} / v_{\mathrm{p}}\right)$, where $v_{0}$ is the laser carrier frequency and $l_{\mathrm{c}}$ the cavity length. If one is interested only in a stable $f_{\text {rep }}$, for example to generate a clock signal in the microwave region, control of $l_{\mathrm{c}}$ seems to be a natural choice and is sufficient. Only when the entire comb spectrum needs to be stabilized do we then need another control freedom, either the pump laser power to influence both $v_{\mathrm{g}}$ and $v_{\mathrm{p}}$, or a swivel mirror reflecting the dispersed spectrum inside the laser cavity to control $v_{\mathrm{g}}$. Therefore, some degree of signal mixing/feedforward is attractive. We would use a fast servo loop acting on $l_{\mathrm{c}}$ to stabilize $f_{\text {rep }}$, with use of the second control loop to influence mainly $f_{\text {ceo }}$. The inevitable variation in $v_{\mathrm{g}}$ caused by the second loop is then compensated for by a properly scaled and opposite-sign feedforward signal to the first loop.

We needed to have two experimental observables to recover the information relating to $f_{\text {rep }}$ and $f_{\text {ceo }}$. We used two beat signals between a cw-stabilized laser and its second harmonic against two respective comb components near the two ends of the comb spectrum. The $\mathrm{cw}$ reference laser was a Nd:YAG laser (frequency $f_{\mathrm{cw}}$ ) with its second harmonic $\left(2 f_{\mathrm{cw}}\right)$ locked on an iodine transition near $532 \mathrm{~nm}$ : this system offered the stability of $5 \times 10^{-14}$ at $1 \mathrm{~s}$ [27]. Both beat signals, $f_{\text {beat } 1064}=n \times f_{\text {rep }}+f_{\text {ceo }}-f_{\text {cw }}$ and $f_{\text {beat } 532}=2 n \times$ $f_{\text {rep }}+f_{\text {ceo }}-2 f_{\mathrm{cw}}$, were recovered with about $30 \mathrm{~dB}$ signal-tonoise ratio at $100 \mathrm{kHz}$ bandwidth. These beat signals were regenerated electronically using the rf tracking-oscillator/filter approach, then mixed in the following way to produce control signals related to $f_{\text {rep }}$ and $f_{\text {ceo }}$, namely $s_{\text {ctrl } 1}=f_{\text {beat532 }}-$ $f_{\text {beat } 1064}=n \times f_{\text {rep }}-f_{\text {cw }}$ and $s_{\text {ctrl2 }}=f_{\text {beat532 }}-2 f_{\text {beat } 1064}=$ $-f_{\text {ceo }}$. Therefore the frequency/phase variations arising in both $f_{\text {rep }}$ and $f_{\text {ceo }}$ were now directly manifested in the two control variables $s_{\text {ctrl1 }}$ and $s_{\text {ctrl2 }}$ and were linked to the optical frequency standard $f_{\mathrm{cw}}$. These two signals could then drive the two servo transducers mentioned above to close the feedback loops.

To demonstrate the effectiveness of our control scheme, we first showed that the stabilization of $f_{\text {rep }}$ was to the optical standard. Essentially we needed to use only the information of $s_{\mathrm{ctrl} 1}$ to control $l_{\mathrm{c}}$ and thus $f_{\text {rep }}$. This approach magnified the noise of $f_{\text {rep }}$ relative to the optical standard by a factor of approximately $3 \times 10^{6}$. In doing so, we could leave the variable $f_{\text {ceo }}$ free-running, since it had been effectively taken out of the control equation. In practice, we used $l_{\mathrm{c}}$ to control the phase of $s_{\mathrm{ctrl} 11}$ to that of another stable oscillator in the rf domain (which translated the optical frequency by a small offset with no degradation of stability). Figure 1 shows the time record of the frequency differences between $f_{\mathrm{cw}}$ and $2813988 \times f_{\text {rep }}$,
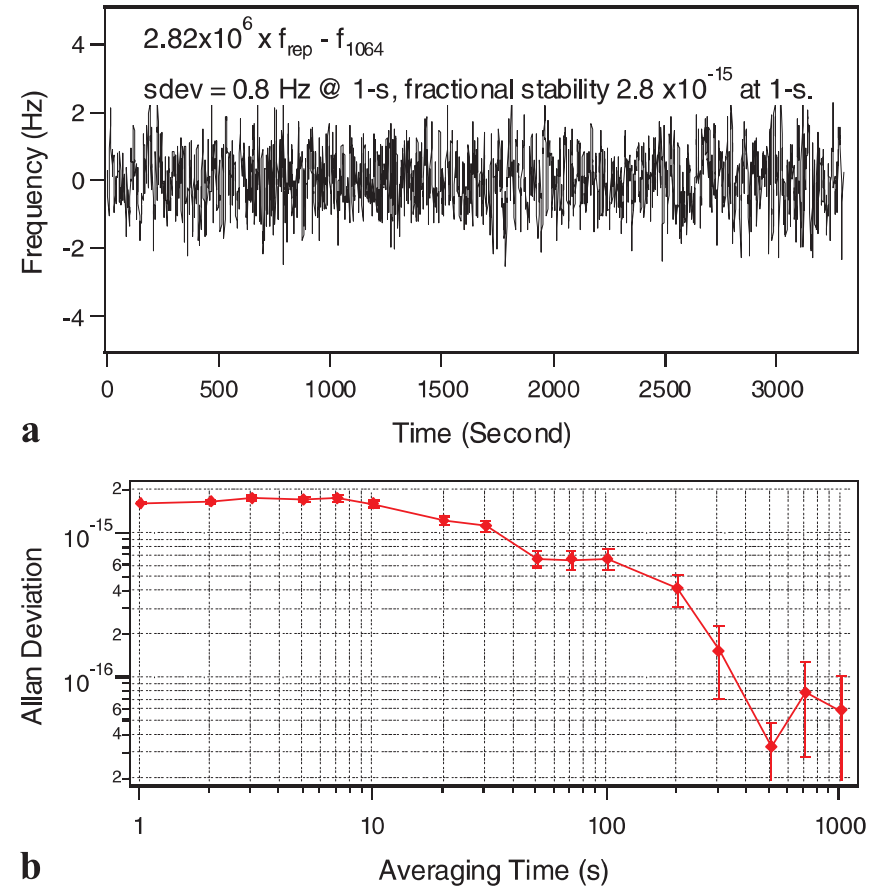

FIGURE 1 Tracking stability of the comb repetition frequency to the cw reference laser. a Time record of the frequency difference between the cw reference laser and the 2.82-millionth harmonic of $f_{\text {rep }}$. b The associated Allan deviation calculated from the time record

with a standard deviation of $0.8 \mathrm{~Hz}$ at a 1-s counter gate time. The Allan deviation calculated from this time record is shown in the bottom trace. The tracking capability of the comb system, at a level of $10^{-15}$ or better, is more than 10-times better than the current optical standard itself.

With the excellent tracking property of the comb system, we expect the stability of the derived clock signal of $f_{\text {rep }}$ to be basically that of the optical standard, namely $5 \times 10^{-14}$ at $1 \mathrm{~s}$. Such an optically derived clock would give its natural time stamps at the $1 / f_{\text {rep }}$ interval and/or its integer multiples. To characterize the system, a reality check would be to compare the optical clock signal against other well-established microwave/rf frequency standards. The international time standard Cs clock should certainly be one of the references; however, the short-term stability of a Cs atomic clock is only approximately $5 \times 10^{-12}$ at $1 \mathrm{~s}$. For improved short-term characterization of the fs comb clock, we also used a hydrogen maser signal transmitted over a 2-km fiber, and another inhouse highly stable crystal oscillator (short-term stability better than $5 \times 10^{-13}$ at $1 \mathrm{~s}$ ), which was slowly slaved to the Cs reference for correcting the frequency offset and drift [28]. Figure 2 summarizes the comparison results of the optical clock against all three rf references. The upper graph shows part of the time record of the beat signal between an $8 \mathrm{GHz}$ synthesized frequency from the crystal oscillator against the 80th harmonic of $f_{\text {rep }}(\sim 100 \mathrm{MHz})$. We used the combination of high harmonic orders and heterodyne beat to help circumvent the resolution limit of the frequency counters. The standard deviation of the beat frequency at 1-s averaging time was $0.0033 \mathrm{~Hz}$. The resultant Allan deviation is shown as the curve in triangles in the bottom graph of the figure. Use of a more stable hydrogen maser signal further reduced the $\mathrm{Al}-$ 

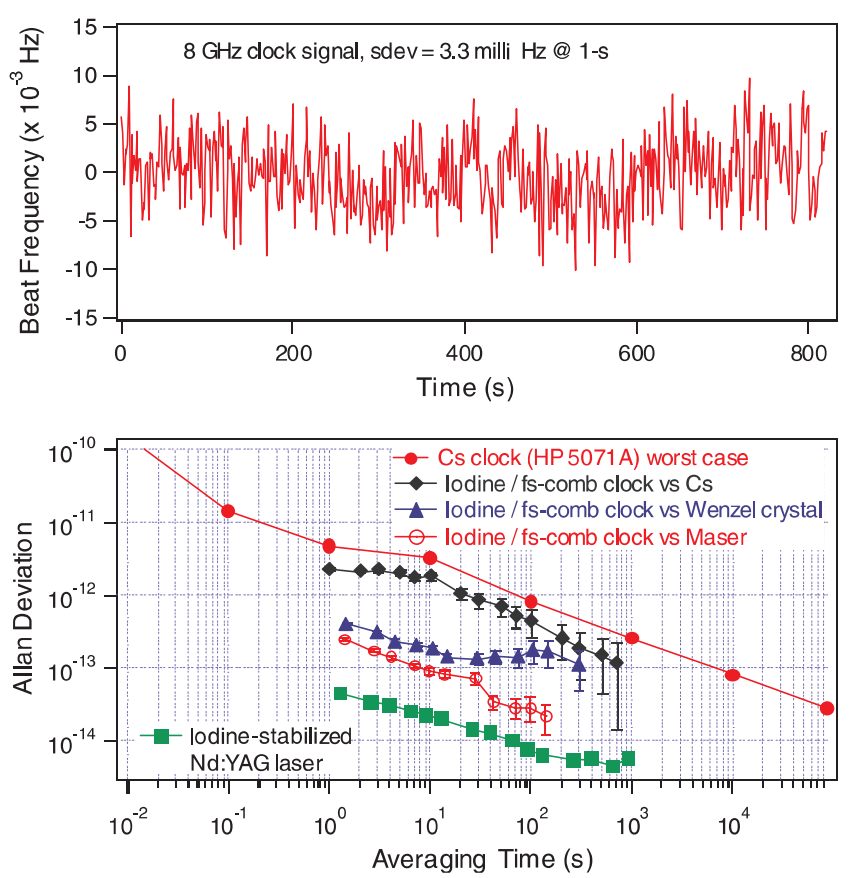

FIGURE 2 Characterization of the clock signal derived from the iodinestabilized laser. The upper graph shows part of the time record of the beat signal between the $8 \mathrm{GHz}$ synthesized frequency of the crystal oscillator referenced to a Cs clock against the 80th harmonic of $f_{\text {rep }}$ of the comb. The lower graph shows the relevant Allan deviations: squares for the iodinestabilized laser; circles for the upper stability limit of the Cs atomic clock; triangles, open circles and diamonds for the beat between the optical clock and the crystal oscillator, the maser and the Cs clock, respectively

lan deviation of the beat, to just below $3 \times 10^{-13}$ at $1 \mathrm{~s}$ (shown with open circles). The beat between the optical and the Cs clock is represented by the curve in diamonds. For comparison, we also display the Allan deviation associated with the Cs atomic clock ("worst case" specification) in circles and the Allan deviation of the iodine-stabilized laser in squares. The data for the optical standard itself were obtained from heterodyne experiments between two similar laser stabilization systems. We note that the superior stability of our optical clock is currently not yet revealed by the microwave-clock-based tests. A microwave source with a better short-term stability can be substantially more expensive, even more than our optical system. Use of two optical clocks would of course be the ultimate choice to perform a thorough cross-check of these new devices. Similar work is being pursued elsewhere [12].

So far, we have made an optical comb that has a welldefined frequency spacing, but the absolute frequencies are uncertain since $f_{\text {ceo }}$ is left floating. An attractive approach to stabilize the entire comb spectrum is to transfer the stability of a single optical standard to the whole set of the comb components throughout the optical bandwidth. To accomplish this task, we needed the information carried by $s_{\text {ctrl2 }}$ to exert a servo action on the comb by the second control parameter, in our case, the swivel mirror. When this second loop was activated, the impact was small on the first loop where $f_{\text {rep }}$ was being stabilized through $l_{\mathrm{c}}$. This was partly due to the fact that the dependence of $f_{\text {rep }}$ and $f_{\text {ceo }}$ on their respective control variables was to a large degree already well separated. The other part of the reason is that fluctuations in $f_{\text {ceo }}$ developed on a slower timescale compared with that of $f_{\text {rep }}$ and therefore a correspondingly slower servo loop was sufficient for stabilization of $f_{\text {ceo }}$. Nevertheless, we took part of the second servo signal and after appropriate signal conditioning we fed this information forward to the first servo loop. The resulting loop performance was improved by about a factor of two (see Fig. 3).

We used the two original optical beats, namely $f_{\text {beat } 1064}$ and $f_{\text {beat532 }}$ that were responsible for generating the control observables but were otherwise outside the servo loops, to characterize the performance of the orthogonal control of the comb. Figure 3 shows the counting record of the two beat frequencies of $f_{\text {beat } 1064}$ and $f_{\text {beat532. Both signals are shown }}$ with their mean values removed but indicated in the figure. Again, the counter gate time was $1 \mathrm{~s}$ and the standard deviations of the two beat signals were $1.7 \mathrm{~Hz}$ for $f_{\text {beat532 }}$ and $1.5 \mathrm{~Hz}$ for $f_{\text {beat } 1064}$. This result indicates that every comb component over the entire optical octave bandwidth was following the $\mathrm{cw}$ laser standard at a level of $3.5 \times 10^{-15}$, again a factor of about 10-times better than the current optical standard itself. The future implication of this work is very clear: With an appropriately chosen optical standard, we can establish an optical frequency grid with lines repeating every $100 \mathrm{MHz}$ over an octave optical bandwidth and with every line stable at the 1-Hz level.

A portable version of this iodine-based optical clock would have a great impact in field applications: we would be able to make precision measurements in length and time with a single device. The frequency stability in both microwave and optical domains is exceptional, surpassing basically all common sources save only national-standards-scale massive installations. The stability $\left(5 \times 10^{-14}\right.$ at $\left.1 \mathrm{~s}\right)$ and reproducibility $\left(4 \times 10^{-13}\right)$ of the $\mathrm{cw}$ laser locked on the iodine transition can be further improved, possibly by another factor of ten. We fully expect such simple optical clock systems will be developed by various interested laboratories.
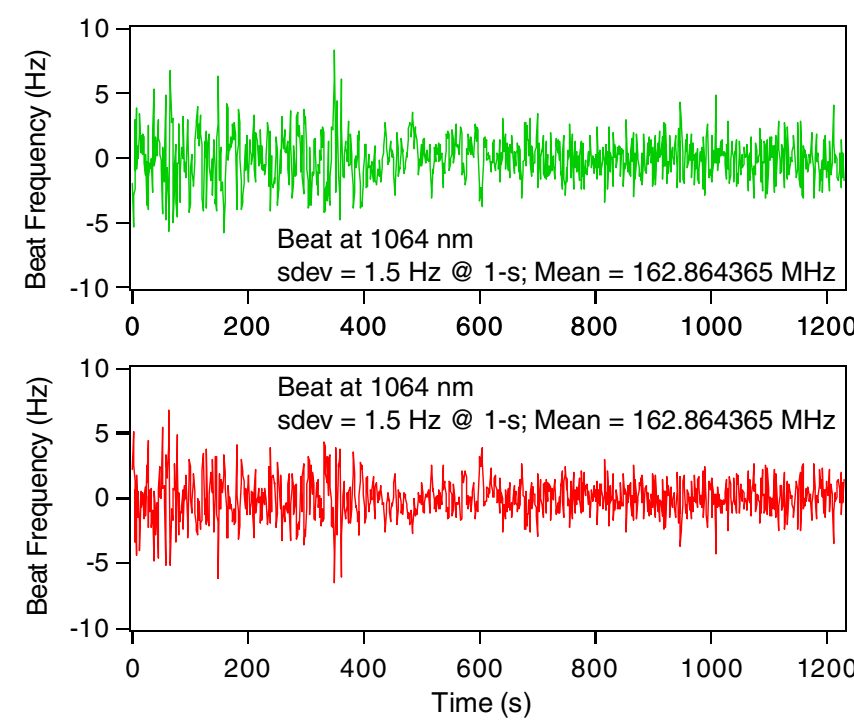

FIGURE 3 Orthogonal control of the entire optical comb, showing Hz-level stability for both beat signals of the cw laser against a comb component at $1064 \mathrm{~nm}$ (bottom trace) and the second harmonic of the cw laser against its corresponding comb line at $532 \mathrm{~nm}$ (upper trace). Better orthogonalization in the control loops leads to reduced noise after $400 \mathrm{~s}$ 
An optical frequency grid with stable lines repeating every $100 \mathrm{MHz}$ over a large optical bandwidth is useful for a number of applications. However, we often desire a single-frequency optical "delta"-function that can be tuned to any preferred frequency position on demand. Realization of such an optical frequency synthesizer (analogous to its radio-frequency counterpart) will add a tremendously useful tool for modern optics-based experiments. One can foresee an array of diode lasers, each covering a successive tuning range of approximately $10-20 \mathrm{~nm}$ and emitting some reasonably useful power, that would collectively cover most parts of the visible spectrum. Each diode laser frequency would be controlled by the stabilized optical comb, and therefore be related to the absolute time/frequency standard, while the setting of the optical frequency would be done through computer control to any desired value. For the first step, we constructed an electronic control system that allowed a widely tunable diode laser to tune through a targeted spectral region at a $10 \mathrm{MHz}$ step while maintaining reference to the stabilized optical comb. A selfadaptive searching algorithm first tuned the laser to within the specified wavelength region with the aid of a wavelength measurement device. The heterodyne beat between the diode laser and the comb lines was then detected and properly processed. An auxiliary rf source provided a tunable frequency offset for the optical beat. Frequency tuning of the diode laser was accomplished by a diode-laser servo control that locked the optical beat to the tunable radio frequency. Once the laser frequency tuning exceeded $100 \mathrm{MHz}$ (going through one comb spacing), we reset the radio frequency offset to the original value to start the process over again. The laser frequency could thus be tuned smoothly in an "inch-worm" fashion through the comb structure [29]. In experiments, we verified this tuning process by using independent optical resonances to monitor the diode-laser frequency. With improved servo bandwidth, the stability of the iodine-stabilized Nd:YAG laser will be faithfully transferred to another $\mathrm{cw}$ laser lying hundreds of THz away.

\section{Carrier-envelope phase stabilization}

Precision control of the time-domain carrierenvelope phase has been a highly desirable and yet elusive goal in ultrafast science since the advent of few-cycle pulses. Normally, the absolute phase of an optical wave's electric field is not relevant, in that any shift in this phase has no measurable effect. However, within the context of few-cycle optical pulses, the electric field does not have this invariance. This condition is due to the reference provided by the few-cycle width of the pulse envelope, and the value of the electric field's phase (relative to the envelope) drastically alters the optical character of a few-cycle pulse. One example of this condition is coherent X-ray (or high-harmonic) generation. In addition to X-ray generation, other examples of extreme non-linear optics or strong field processes where the absolute phase is critical include: attosecond-pulse generation and strong-field ionization.

Frequency-domain-based laser control techniques have had a profound impact on time-domain carrier-envelope phase stabilization. The first experimental demonstration of pulse-to-pulse carrier-envelope phase control $[14,15]$ quickly followed the initial proposal $[30,31]$. In general, the absolute carrier-envelope phase, $\phi$, of the $m$ th pulse is given by $\phi_{m}=\phi_{0}+m \Delta \phi$ where $\phi_{0}$ is an unknown constant phase offset and $m$ is an integer labeling individual pulses. As already described, the quantity $\Delta \phi$ is the pulse-to-pulse phase slip, which is related to the offset frequency and pulse repetition rate via $\Delta \phi=2 \pi f_{\text {ceo }} / f_{\text {rep }}$, or equivalently, $\Delta \phi=\left(\frac{1}{v_{\mathrm{g}}}-\frac{1}{v_{\mathrm{p}}}\right) l_{\mathrm{c}} 2 \pi v_{0} \bmod 2 \pi$. Therefore, control of $\Delta \phi$ requires the maintenance of a stable ratio between $f_{\text {ceo }}$ and $f_{\text {rep. }}$. It is straightforward to measure $f_{\text {ceo }}$ when the bandwidth of the frequency comb spans an optical octave. Basically, we measured the heterodyne beat between comb lines at the highfrequency extreme of the spectrum and the second harmonic of the corresponding comb lines at the low-frequency end of the spectrum. The resulting beat frequency was given by $\left|f_{2 n}-2 f_{n}\right|=\left|\left(f_{\text {ceo }}+2 n f_{\text {rep }}\right)-2\left(f_{\text {ceo }}+n f_{\text {rep }}\right)\right|=f_{\text {ceo }}$.

Once $f_{\text {ceo }}$ was measured, its stabilization was enabled through control of the pulse group delay in the cavity, again implemented with a swivel-end mirror behind the dispersion compensation prism pair. For this application, we were interested in the control of the ratio $f_{\text {ceo }} / f_{\text {rep }}$. For example, by setting $f_{\text {ceo }}$ to be $(N / 16) f_{\text {rep }}$, where $N$ is an integer between 1 to 16 , we could lock the pulse-to-pulse carrier-envelope phase slip, $\Delta \phi=2 \pi N / 16$, at 16 different positions between 0 and $2 \pi$. For example, if $N=4$, then every fourth pulse had the same phase. Control of $\Delta \phi$ was verified in the time domain by second-order interferometric cross-correlation. Correlation of pulse $i$ with pulse $i+2$ was achieved using a multi-pass cell for the $20 \mathrm{~ns}$ delay needed to correlate one pulse with a second pulse, two cavity round-trips later. A typical cross-correlation is shown in Fig. 4a. To determine the pulse-to-pulse phase slip, the fringe peaks of a correlation were fit to a Gaussian envelope. In a cross-correlation, the phase difference between the electric fields of the two pulses results in a shift in the peaks of the fringes with respect to the envelope. A plot of
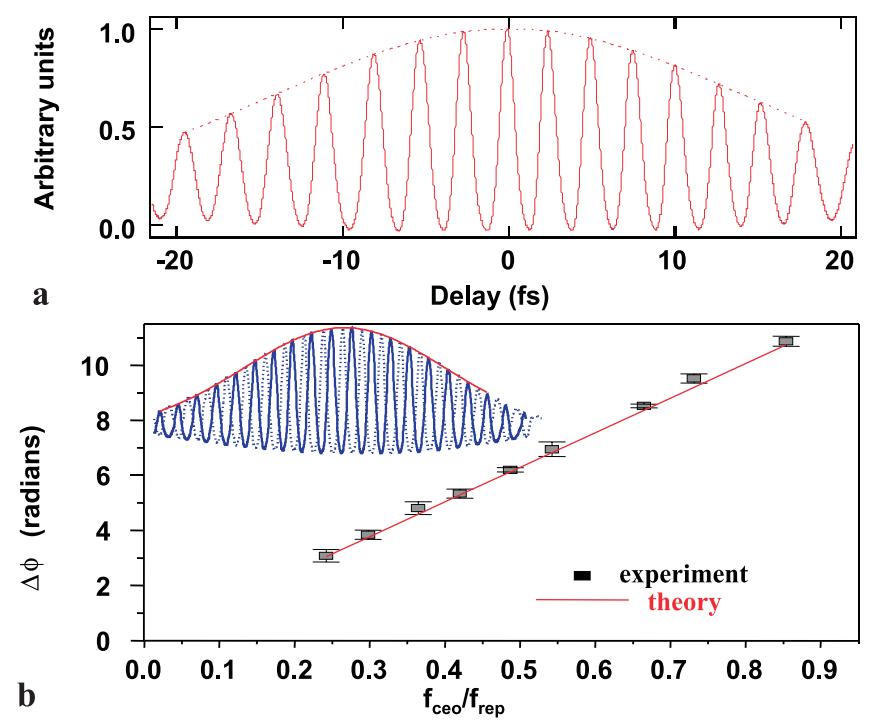

FIGURE 4 a Typical cross-correlation, solid line, and the fit to a Gaussian envelope, dashed line; and $\mathbf{b}$ experimentally determined values of pulseto-pulse carrier-envelope phase change for different frequency offsets and a linear fit to the results. The inset shows two correlations with a controlled $\pi$ phase shift 
experimentally determined values of relative phase for different frequency offsets, $f_{\text {ceo }}=(N / 16) f_{\text {rep }}$, and a linear fit to the results is given in Fig. $4 \mathrm{~b}$. The results show a good agreement with the theoretically expected relation $2 \Delta \phi=2 \times$ $2 \pi f_{\text {ceo }} / f_{\text {rep }}$. Also, the precision to which we could stabilize $f_{\text {ceo }}$ told us that we should be able to stabilize $\Delta \phi$ to less than $1 \mu \mathrm{rad}$.

An important detail is that in all these cases we have fixed the relative pulse-to-pulse phase slip, not the overall "absolute" phase of each pulse, i.e. $\phi_{m}$ (which includes $\phi_{o}$ ). Control of the absolute phase of a short pulse is of interest to the ultrafast community. Recently, the development of few-cycle pulses allows for the possibility of extreme non-linear optical processes such as X-ray generation and above-threshold ionization, the efficiencies of which are highly dependent on the peak electric field intensity. Vice versa, experiments have been proposed to take advantage of a highly non-linear process with such dependence to determine the absolute phase.

For future extreme non-linear optics experiments employing amplifier-based ultrashort pulses, control of the carrierenvelope phase will be a more complicated problem [32-34]. In the work described above, the relevant quantity is the pulseto-pulse phase change $\Delta \phi$, which occurs on a timescale of $10 \mathrm{~ns}$ for a $100-\mathrm{MHz}$-repetition-rate laser. The excursion of $\Delta \phi$ at this short timescale has been proved to be very small, of the order of nanoradians, even with a free-running laser. The feedback loop that controls $\Delta \phi$ has a typical servo bandwidth of approximately $1 \mathrm{kHz}$, which due to its phase-lock nature is able to hold the mean value of $\Delta \phi$ constant without any longterm drift. With the r.m.s. excursion small and the mean value being held constant, interference signals of many pulse pairs can coherently add up, without a diminishing contrast. However, if one wants to measure the r.m.s. phase noise of $\Delta \phi_{N}$, meaning the change of carrier envelope phase between pulse $i$ and pulse $i+N$, then we can naively expect $\Delta \phi_{N}=2 \pi f_{\text {ceo }} \times$ $N T=2 \pi \times f_{\text {ceo }} /\left(f_{\text {rep }} / N\right)$. From this we know that we will not get a $\Delta \phi_{N}$ that has a phase jitter of less than $2 \pi$. The reason is that $f_{\text {ceo }}$ has a few $\mathrm{kHz}$ r.m.s. noise at millisecond timescales.

A more rigorous treatment requires measurement of the r.m.s. phase noise of $f_{\text {ceo }}$ itself. The reason for the r.m.s. phase noise of $f_{\text {ceo }}$ being equivalent to the carrier-envelope phase noise is that we can interpret $\Delta \phi=2 \pi\left(f_{\text {ceo }} / f_{\text {rep }}\right)$ simply as the phase accumulation in a measurement time window of $\left(1 / f_{\text {rep }}\right)$ when $f_{\text {ceo }}$ is not zero. The noise power spectral density of frequency $\left(s_{\nu}^{f_{\text {ceo }}}\right)$ and phase $\left(s_{\phi}^{f_{\text {ceo }}}\right)$ are related by $s_{\phi}^{f_{\text {ceo }}}=s_{\nu}^{f_{\text {ceo }}} / f^{2}$. Within a time window of $\tau_{\text {obs }}$, we determine the phase noise accumulation due to a non-zero $f_{\text {ceo }}$ as $\left.\Delta \phi_{\mathrm{rms}}\right|_{\tau_{\mathrm{obs}}}=\sqrt{\int_{1 / 2 \pi \tau_{\mathrm{obs}}}^{\infty} \frac{1}{f^{2}} s_{\nu}^{f_{\mathrm{ceo}}}(f) \mathrm{d} f}$.

Careful design of a sufficiently fast servo loop along with stable system (oscillator plus amplifier) construction will help to achieve the goal of $\left.\Delta \phi_{\mathrm{rms}}\right|_{\mathrm{ms} \sim \mathrm{s}}<\pi$.

\section{Synchronization and phase-lock of independent femtosecond lasers}

Our motivation for working with separate ultrafast lasers stems from the desire to create an arbitrary light- waveform generator, with the capability of synchronizing and phase-locking arbitrary, separate mode-locked lasers of distinct optical properties. The ability to generate coherent light with ultrabroad bandwidths is essential for many applications in ultrafast science and technology. In the past 10 years, advances in this area have led to broad-bandwidth, ultrashort-pulse laser systems [3] that routinely generate sub$10 \mathrm{fs}$ pulses [35]. Moreover, coherent bandwidths can be extended over very broad spectral regions using non-linear frequency conversion techniques such as white-light continuum generation [36], parametric amplification [37], molecular phase modulation [38] etc. However, for some applications, these techniques can suffer from poor efficiency and lack of flexibility, in particular for applications where arbitrary pulse synthesis at very different wavelengths is required. Synthesis of arbitrary pulses by coherently combining the output fields of two or more separate lasers would enable the generation of optical waveforms with distinct optical properties in distinct regions of the spectrum with potentially high powers.

Phase-locking of separate femtosecond (fs) lasers, however, requires both tight synchronization between the two pulse trains as well as effective detection of the phase difference between the two optical carrier waves underlying the pulse envelopes. As a first step, we synchronized two Kerrlens mode-locked (KLM) fs lasers by controlling the repetition rates of the two lasers to be identical to a very high degree of precision $\left(f_{\text {rep } 1}=f_{\text {rep } 2}\right)$. This was achieved via direct phase-locking of the repetition frequencies of the lasers. The next step was to ensure that the spectral combs of the individual lasers were exactly coincident in the region of spectral overlap so that the two sets of optical frequency combs formed a continuous and phase-coherent entity. We detected a coherent heterodyne beat signal between the corresponding comb components of the two lasers. Such heterodyne detection yields information related to $\left(f_{\mathrm{ceo} 1}-f_{\mathrm{ceo} 2}\right)$, which can then be controlled. By phase-locking $\left(f_{\text {ceol } 1}-f_{\text {ceo } 2}\right)$ to a frequency of mean value zero, we effectively demanded that $\left(\Delta \phi_{1}-\Delta \phi_{2}\right)=0$, leading to two pulse trains that had nearly identical dynamic evolution.

The two independent mode-locked Ti:sapphire lasers [4] each operated at a $100 \mathrm{MHz}$ repetition rate, with one centered at $760 \mathrm{~nm}$ and the other at $810 \mathrm{~nm}$. The bandwidth of each laser corresponded to a sub-20 fs transform-limited pulse. Our synchronization scheme employed two phase-locked loops (PLL) working at different time resolution. The first loop operated at the fundamental repetition frequency to provide a full dynamic range of timing offset, while the second operated at the 80th harmonic of $f_{\text {rep }}(8 \mathrm{GHz})$ to provide enhanced phase stability. Using this approach, we have demonstrated the timing jitter between the two separate pulse trains to be $<5 \mathrm{fs}$, at a bandwidth of $160 \mathrm{~Hz}$, observed over several minutes [16]. The flexibility of this synchronization system was demonstrated by locking two independent lasers working at different commensurable repetition frequencies.

Under synchronization, the heterodyne beat between the two corresponding sets of combs could be recovered with a signal-to-noise ratio $(S / N)$ of $60 \mathrm{~dB}$ in a $100 \mathrm{kHz}$ bandwidth. Phase shift in the synchronization lock loop was adjusted to have the two pulses optimally overlapped tempo- 
rally at the heterodyne detector to produce the maximum beat signal. Hundreds of comb pairs contributed to the heterodyne beat signal, and its amplitude was coherently enhanced when the synchronization was effective. By stabilizing $\left(f_{\text {ceo } 1}-f_{\text {ceo } 2}\right)$ to a mean value of $0 \mathrm{~Hz}$, the carrierenvelope phase slip per pulse of one laser was accurately matched by the second laser. Locking of this beat frequency to $0 \mathrm{~Hz}$ was implemented using an acousto-optic modulator (AOM). One laser beam passed through the AOM and was offset by the drive frequency of the AOM. This avoided the need to process the beat signal in the troublesome frequency range around $0 \mathrm{~Hz}$. The beat was then phase-locked to the drive frequency of the AOM, effectively removing the AOM frequency from the beat.

The carrier frequency beat signal between the two lasers was recorded under phase-locked conditions, with a standard deviation of $0.15 \mathrm{~Hz}$ at 1 -s averaging time. In contrast, without phase-locking, the beat frequency between the two synchronized lasers had a standard deviation of a few MHz. We also performed a direct time-domain analysis of the coherence between the two fs lasers. Spectral interferometry analysis was carried out by combining the two laser pulses on a beam splitter and directing the two pulses collinearly into a spectrometer that consisted of a grating and a CCD array. The spectrum of the sum of the two pulses produced the phase difference between the two pulses, and a preset delay $\tau$ between the two pulses yielded fringes in the combined spectrum if coherence between the two pulse trains persisted over the measurement time period (typically a few $\mu$ s to a few ms). The combined spectrum was smooth when the two lasers were not phaselocked. Under phase-locked conditions, interference fringes were produced, which revealed excellent phase coherence between the two pulses within the observation time.

To demonstrate the "coherently synthesized" aspect of the combined pulse, a second-order autocorrelation measurement of the combined pulse was carried out. For this measurement, the two pulse trains were maximally overlapped in the time domain before the autocorrelator. One arm of the autocorrelator (Michelson interferometer) was scanned to collect the autocorrelation data. The second-order autocorrelation curves of each individual laser are shown (Fig. 5A and B, respectively). The spectrum of one laser was centered around $760 \mathrm{~nm}$ while that for the other was around $810 \mathrm{~nm}$. Under phase-lock, the spectral interferometry measurement displayed clear interference fringes within the overlapping spectra (Fig. 5C). The fringe visibility was reduced when the measurement time was increased, due to the increased phase noise between the two lasers at longer observation times. An interesting autocorrelation measurement was shown when the two lasers were not even synchronized (Fig. 5D). Basically, we obtained an autocorrelation of a single laser pulse, with a sharp spike appearing in the data at a random position. The spike appeared because, at that particular instant, the pulses overlapped in time and the two electric fields came into phase and coherently added together. The timescale of this random interference was related to the offset frequency between the two repetition rates and was usually less than a few nanoseconds. When the two lasers were synchronized but not phase-locked, the resulting autocorrelation measurement indicated an increased signal amplitude compared to the unsynchronized case, typically by a factor of 2.7 (Fig. 5E). However, as expected this signal displayed considerable random phase noise within the
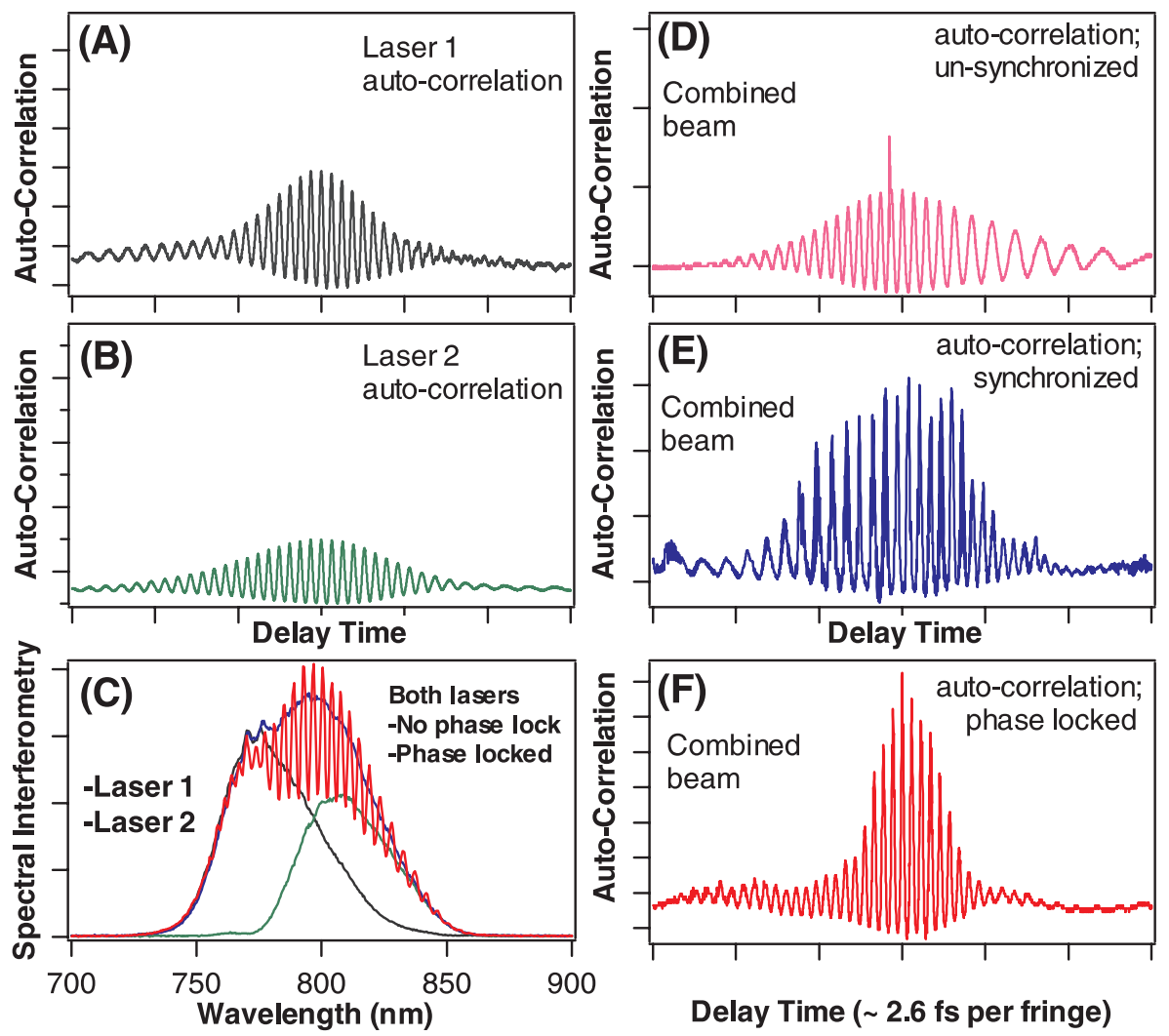

FIGURE 5 Second-order autocorrelation measurement of the combined pulse. Secondorder auto-correlation data of the two individual lasers are shown in (A) and (B). In (C), the spectral interferometry data indicate that the spectra of the two lasers are separated, with interference fringes clearly present in the overlap region of the two spectra. The pulse trains from the two lasers are combined and sent to a collinear autocorrelator. Data in (D) represent the case when the two lasers are not synchronized. The two fields are in phase only briefly, resulting in a random sharp spike in the autocorrelation curve. Data in (E) are obtained when the two lasers are synchronized but not phase-locked. The increase in the autocorrelation amplitude is about 2.7-times that for the previous unsynchronized case. When the two lasers are both synchronized and phase-locked, the second-order autocorrelation data in $(\mathrm{F})$ show the pulse width is narrowed and pulse amplitude increased by $>20 \%$. Notice the large difference in noise between the phase-locked and unlocked autocorrelation curves 
autocorrelation interference fringes. When the two fs lasers were phase-locked, the autocorrelation revealed a clean pulse that was often shorter in apparent duration and larger in amplitude (Fig. 5F). This result is statistically significant because this pulse shortening was observed in more than $40 \%$ of the total data scans. Note however, that in this work we did not attempt to recompress the light pulses outside the laser cavities to very short duration, and the pulses were dispersively broadened to 50-70 fs in duration. The width of the central fringe pattern in an interferometric autocorrelation is more a characteristic of the overall bandwidth of the pulse than of the pulse duration, and can result in a trace that appears deceptively short when the overall time span of the scan is short. However, the data clearly show the difference between a coherently phase-locked pulse train (Fig. 5F) and one with a complex and random frequency sub-structure (Fig. 5E). The averaging of hundreds of the autocorrelation scans also consistently showed an amplitude enhancement by more than $20 \%$ when the phase-lock was activated. We have therefore demonstrated a successful implementation of coherent light synthesis: the coherent combination of the outputs from more than one laser in such a way that the combined output can be viewed as coherent, femtosecond pulse being emitted from a single source [17].

An important issue in the future is to demonstrate control over the phase profile across the entire synthesized spectrum, namely pulse shaping. For example, a flat spectral phase profile is a prerequisite for generating an ultrashort pulse, while arbitrary shape is needed for coherent control applications. Using the current control scheme, the carrier-envelope phase slips of the two lasers track each other $\left(\Delta \phi_{\mathrm{x}}=\Delta \phi_{\mathrm{y}}\right)$. There remains of course a static phase difference between the two lasers, namely $\left(\phi_{\mathrm{x}}-\phi_{\mathrm{y}}\right)$. However, this static phase can be controlled through an appropriate phase offset introduced in the carrier heterodyne beat detection, for example, through phase shift of the rf signal driving the AOM. This phasecompensated spectrum can then be used in a pulse-shaping device to generate the desired pulse shape.

ACKNOWLEDGEMENTS We thank S. Diddams, L. Hollberg, J. Bergquist, J. Kitchin, R. Bartels, T. Weinacht, S. Backus, D. Anderson, J. Levine and R. Windeler for useful discussions. The work at JILA is supported by NASA, NIST, NSF and the Research Corporation. JY, STC and JLH are staff members of the Quantum Physics Division of NIST Boulder. KWH acknowledges financial support from the Fannie and John Hertz Foundation.

\section{REFERENCES}

1 J.N. Eckstein, A.I. Ferguson, T.W. Hänsch: Phys. Rev. Lett. 40, 847 (1978)

2 E.V. Baklanov, V.P. Chebotaev: Soviet J. Quant. Electron. 7, 1252 (1977)

3 D.E. Spence, P.N. Kean, W. Sibbett: Opt. Lett. 16, 42 (1991)

4 M.T. Asaki, C.P. Huang, D. Garvey, J.P. Zhou, H.C. Kapteyn, M.M. Murnane: Opt. Lett. 18, 977 (1993)

5 T.A. Birks, J.C. Knight, P.S. Russell: Opt. Lett. 22, 961 (1997)
6 J.K. Ranka, R.S. Windeler, A.J. Stentz: Opt. Lett. 25, 25 (2000)

7 T. Udem, J. Reichert, R. Holzwarth, T.W. Hänsch: Phys. Rev. Lett. 82, 3568 (1999)

8 S.A. Diddams, D.J. Jones, J. Ye, T. Cundiff, J.L. Hall, J.K. Ranka, R.S. Windeler, R. Holzwarth, T. Udem, T.W. Hänsch: Phys. Rev. Lett. 84, $5102(2000)$

9 M. Niering, R. Holzwarth, J. Reichert, P. Pokasov, T. Udem, M. Weitz, T.W. Hänsch, P. Lemonde, G. Santarelli, M. Abgrall, P. Laurent, C. Salomon, A. Clairon: Phys. Rev. Lett. 84, 5496 (2000)

10 J. Ye, T.H. Yoon, J.L. Hall, A.A. Madej, J.E. Bernard, K.J. Siemsen, L. Marmet, J.M. Chartier, A. Chariter: Phys. Rev. Lett. 85, 3797 (2000)

11 T. Udem, S.A. Diddams, K.R. Vogel, C.W. Oates, E.A. Curtis, W.D. Lee, W.M. Itano, R.E. Drullinger, J.C. Bergquist, L. Hollberg: Phys. Rev. Lett. 86, 4996 (2001)

12 S.A. Diddams, T. Udem, J.C. Bergquist, E.A. Curtis, R.E. Drullinger, L. Hollberg, W.M. Itano, W.D. Lee, C.W. Oates, K.R. Vogel, D.J. Wineland: Science 293, 825 (2001)

13 J. Ye, L.S. Ma, J.L. Hall: Phys. Rev. Lett. 87, 270801 (2001)

14 D.J. Jones, S.A. Diddams, J.K. Ranka, A. Stentz, R.S. Windeler, J.L. Hall, S.T. Cundiff: Science 288, 635 (2000)

15 A. Apolonski, A. Poppe, G. Tempea, C. Spielmann, T. Udem, R. Holzwarth, T.W. Hänsch, F. Krausz: Phys. Rev. Lett. 85, 740 (2000)

16 L.S. Ma, R.K. Shelton, H.C. Kapteyn, M.M. Murnane, J. Ye: Phys. Rev. A 64, 021802 (2001)

17 R.K. Shelton, L.S. Ma, H.C. Kapteyn, M.M. Murnane, J.L. Hall, J. Ye: Science 293, 1286 (2001)

18 A.M. Weiner, D.E. Leaird, G.P. Wiederrecht, K.A. Nelson: Science 247, $1317(1990)$

19 K.W. Delong, R. Trebino, J. Hunter, W.E. White: J. Opt. Soc. Am. B 11, 2206 (1994)

20 C. Iaconis, I.A. Walmsley: Opt. Lett. 23, 792 (1998)

21 T. Brabec, F. Krausz: Rev. Mod. Phys. 72, 545 (2000)

22 S.T. Cundiff, J. Ye, J.L. Hall: Rev. Sci. Instrum. 72, 3749 (2001)

23 J. Ye, J.L. Hall, S.A. Diddams: Opt. Lett. 25, 1675 (2000)

24 L. Hollberg, C.W. Oates, E.A. Curtis, E.N. Ivanov, S.A. Diddams, T. Udem, H.G. Robinson, J.C. Bergquist, R.J. Rafac, W.M. Itano, R.E. Drullinger, D.J. Wineland: IEEE J. Quantum Electron. QE-37, $1502(2001)$

25 R.J. Rafac, B.C. Young, J.A. Beall, W.M. Itano, D.J. Wineland, J.C. Bergquist: Phys. Rev. Lett. 85, 2462 (2000)

26 J.L. Hall, L.S. Ma, M. Taubman, B. Tiemann, F.L. Hong, O. Pfister, J. Ye: IEEE Trans. Instrum. Meas. 48, 583 (1999)

27 J. Ye, L. Robertsson, S. Picard, L.S. Ma, J.L. Hall: IEEE Trans. Instrum. Meas. 48, 544 (1999)

28 Cs clock: HP 5071 A; Crystal oscillator: Wenzel 5/10 Blue Top Ultra Low Noise Oscillator; Hydrogen maser: ST-22, Clock-13, NIST, Boulder. We thank our NIST colleagues for the fiber-based delivery of the maser signal to JILA. Mentioning of commercial products is for technical communications only

29 J.D. Jost, J.L. Hall, J. Ye: submitted for publication (2002)

30 J. Reichert, R. Holzwarth, T. Udem, T.W. Hänsch: Opt. Commun. 172, 59 (1999)

31 H.R. Telle, G. Steinmeyer, A.E. Dunlop, J. Stenger, D.H. Sutter, U. Keller: Appl. Phys. B 69, 327 (1999)

32 M. Kakehata, H. Takada, Y. Kobayashi, K. Torizuka, Y. Fujihira, T. Homma, H. Takahashi: Opt. Lett. 26, 1436 (2001)

33 A. Poppe, R. Holzwarth, A. Apolonski, G. Tempea, C. Spielmann, T.W. Hänsch, F. Krausz: Appl. Phys. B 72, 373 (2001)

34 F.W. Helbing, G. Steinmeyer, U. Keller, R.S. Windeler, J. Stenger, H.R. Telle: Opt. Lett. 27, 194 (2002)

35 J.P. Zhou, G. Taft, C.P. Huang, M.M. Murnane, H.C. Kapteyn, I.P. Christov: Opt. Lett. 19, 1149 (1994)

36 R.R. Alfano: The Supercontinuum Laser Source (Springer, New York 1989)

37 P.B. Corkum, N.H. Burnett, M.Y. Ivanov: Opt. Lett. 19, 1870 (1994)

38 A.V. Sokolov, D.R. Walker, D.D. Yavuz, G.Y. Yin, S.E. Harris: Phys. Rev. Lett. 85, 562 (2000) 